\title{
New Hybrid Technology for Planar Fluxgate Sensor Fabrication
}

\author{
Olivier Dezuari, Eric Belloy, Scott E. Gilbert, and Martin A. M. Gijs
}

\begin{abstract}
We have adapted a new printed circuit board (PCB) technology to the fabrication of ultraflat and sensitive fluxgate magnetic field sensors. The two outer layers of the PCB stack compose the electrical windings of fluxgates, while the inner layer is made of a micro-patterned amorphous magnetic ribbon with extremely high relative magnetic permeability $\left(\mu_{r} \approx 100000\right)$. Two basic configurations were considered: one based on a toroidal magnetic core and the other on a rectangular core with and without an air gap. The field response and sensitivity of the fluxgate devices have been studied as a function of the gap length, the excitation current, and excitation frequency. Compared to fluxgate sensors of similar size, a relatively high sensitivity of $60 \mathrm{~V} / \mathrm{T}$ was found at $30 \mathrm{kHz}$ for a five-winding detection coil wound around a rectangular E-shaped magnetic core. This high performance is primarily attributable to the high-permeability magnetic core. The results clearly show the potential of this fluxgate device for application as a magnetic sensor.
\end{abstract}

\section{INTRODUCTION}

$\mathbf{T}$ HANKS to the advanced development of microfabrication technologies during recent years, many sensor operation principles have been transferred from the "macroscopic" world to miniaturized and integrated versions; these often have shown improved properties with respect to the macroscopic devices. An example of such device for the sensitive detection of magnetic fields is the magnetic fluxgate sensor [1]-[3]. A conventional fluxgate sensor usually has a magnetic bulk or ribbon core and mechanically wound wires for realizing the excitation and detection coils. The device is mounted on a printed circuit board (PCB), on which also the electronic circuitry and IC's for the signal processing are attached. The result is a system with fairly large volume, weight, and fabrication costs. Recently, several fluxgate sensors have been demonstrated, which were directly integrated on $\mathrm{Si}$ wafers along with their signal processing circuitry. In these applications, the magnetic material, which is at the heart of the fluxgate operation, is deposited using a vacuum deposition method or by electroplating technologies directly on $\mathrm{Si}$.

The principle of a fluxgate is the external magnetic fielddependent periodic saturation of a ferromagnetic material. Soft magnetic alloys like $\mathrm{Ni}_{80} \mathrm{Fe}_{20}$ (permalloy), having a small hysteresis and a large saturation magnetization, are well known for their use as core material. The combined action of the external field (to be measured) and excitation coils, driving the ferro-

Manuscript received June 16, 1998; revised February 19, 1999.

The authors are with the Institute of Microsystems, Swiss Federal Institute of Technology CH-1015 Lausanne, Switzerland (e-mail: scott.gilbert@epfl.ch; martin.gijs@epfl.ch).

Publisher Item Identifier S 0018-9464(99)04902-X. magnetic core in saturation, leads to the generation of higher order harmonics of the fundamental excitation frequency in a detection coil surrounding the magnetic core. The second harmonic voltage $V_{2 f}$ can be filtered out of the detection signal using a lock-in technique and is proportional to the external field for a certain field range (typically 0.01-100 $\mu \mathrm{T}$ ).

Kawahito et al. [4] have fabricated fluxgate sensors with a size of a few $\mathrm{mm}$ on $\mathrm{Si}$ wafers; they used a multimask technology based on electroplated permalloy cores and microstructured solenoids for the excitation and detection coils. They achieved a sensitivity of $5.8 \mathrm{~V} / \mathrm{T}$ at $100 \mathrm{kHz}$ and 0.58 $\mathrm{V} / \mathrm{T}$ at $10 \mathrm{kHz}$ excitation current signals. The excitation current was $130 \mathrm{~mA}_{\mathrm{p}-\mathrm{p}}$.

Seitz [5] proposed a device based on the integration of a planar fluxgate element and a spiral pick-up coil. The excitation coil was not integrated on the Si chip. Choi et al. [6] demonstrated later a permalloy based micro-fluxgate magnetic sensor using both integrated planar excitation and detection coils combined with on-chip sensor interface circuits and electronics. The fabricated planar sensing element had a magnetic sensitivity of $73 \mathrm{~V} / \mathrm{T}$ at $1 \mathrm{MHz}$ excitation frequency and with a $150 \mathrm{~mA}_{\mathrm{p}-\mathrm{p}}$ excitation current. The same device, when operated with the integrated driver electronics, showed a sensitivity of $90 \mathrm{~V} / \mathrm{T}$ at $610 \mathrm{kHz}$ excitation frequency and 110 mAp-p excitation current.

Kawahito et al. [7] have shown how an optimal coupling between the excitation and pick-up coils and an optimized fabrication of the soft magnetic films can give rise to a sensitivity of $2700 \mathrm{~V} / \mathrm{T}$ at excitation frequencies of $3 \mathrm{MHz}$ and excitation currents of the order of $100 \mathrm{~mA}$. Gottfried-Gottfried et al. [8] also have combined a planar technology for the fluxgate with a CMOS-ASIC for sensor supply, readout, and signal processing. The system exhibits a sensitivity of $9200 \mathrm{~V} / \mathrm{T}$ at an excitation frequency of $350 \mathrm{kHz}$ and for 32 $\mathrm{mA}$ supply currents for the complete magnetic measurement system.

In contrast to previous work, which is based on $\mathrm{Si}$ microfabrication technology, this paper describes a new and inexpensive hybrid PCB-like technology for the realization of fluxgate magnetic sensors. Recent developments in the area of ferromagnetic magnetoresistive field sensors have led to devices with sensitivities on the order of $100 \mathrm{~V} / \mathrm{T}$ [9], competing with miniature fluxgate sensors. However, in comparison to magnetoresistive sensors, fluxgate-based magnetic field sensors have the potential advantages of low noise, and when employed in negative feedback configurations, exhibit high linearity and high temperature stability. 
The fluxgate sensors described herein are based on two geometries; the magnetic cores have either toroidal or rectangular shapes. These devices are built up of three layers of which the outer layers carry the $\mathrm{Cu}$ winding coil patterns and the inner layer is a patterned high permeability amorphous ferromagnetic sheet core. For this work, a particular alloy, known under the trade name Vitrovac $\AA$ 6025, (composition $\left.(\mathrm{Co}, \mathrm{Fe}, \mathrm{Mo})_{73}(\mathrm{Si}, \mathrm{B})_{27}\right)$, was chosen primarily because of its extremely high relative permeability $\left(\mu_{r} \approx 100000\right) .{ }^{1}$ Both magnetic metal and copper layers are patterned using standard lithographic techniques. In between the magnetic metal and copper layers there is a foil of solid epoxy glue (Prepreg ${ }^{\circledR}$, typically $100 \mu \mathrm{m}$ thick) for insulation and assembly. Such material is well known in the assembly of multilayer PCB structures. Connection between the outer copper patterns is realized by electroplating methods to complete the windings. These fluxgate devices are about $600 \mu \mathrm{m}$ thick, with lateral dimensions of approximately $1 \mathrm{~cm}$.

\section{FluXgate Design}

Two different types of fluxgate sensors have been designed and fabricated: the first type has the inner magnetic foil patterned into a toroidal shape and the second type has a magnetic core of rectangular shape. Diagrams of these two structures are displayed in Fig. 1(a) and (b). One should note that in Fig. 1(a), the two excitation coils are positioned symmetrically at both sides of the detection coil. Each excitation coil has 18 windings while only four were used for the detection coil.

Fig. 1(b) shows a schematic diagram of a rectangular fluxgate sensor whose excitation coils have been grouped into four clusters of five winding and are symmetrically positioned around the inner five detection coil windings. Moreover, we have introduced air gaps in the magnetic core. A series of rectangular fluxgate devices with gap lengths $L_{g}$ varying between 0 and $1 \mathrm{~mm}$ were fabricated to investigate their influence on the sensitivity and the linear range of such a magnetic sensor, as will be discussed below. Photographs of the experimental realization of the two types of fluxgate sensors are displayed in Fig. 2.

\section{FABRICATION PROCESS}

A schematic diagram of the coil fabrication process is shown in Fig. 3. The proposed device fabrication is very similar to a conventional PCB process. These devices are based on two epoxy boards (100 $\mu$ m thick) with copper ( $35 \mu \mathrm{m}$ thick) laminated on one side, and by a simple epoxy board $(100 \mu \mathrm{m}$ thick) as support for the Vitrovac ${ }^{\circledR}$ magnetic foil. A liquid epoxy was used to laminate the magnetic foil on the inner epoxy board. Two $100 \mu \mathrm{m}$-thick sheets of Prepreg ${ }^{\circledR}$ solid epoxy were used for bonding the laminates into a stack of five layers.

The Vitrovac ${ }^{\circledR} 6025$ foil was patterned photolithographically to form the fluxgate cores by first laminating sheets of solid negative photoresist $\left(\operatorname{Ordyl}{ }^{\circledR} 200\right)^{2}$ onto degreased foils

\footnotetext{
${ }^{1}$ Vitrovac ${ }^{\circledR}$ product literature, Vacuumschmelze GmbH, Hanau, Germany.

${ }^{2}$ Ordyl® AF 200 product literature, Elga Ronal SRL., Milan, Italy.
}

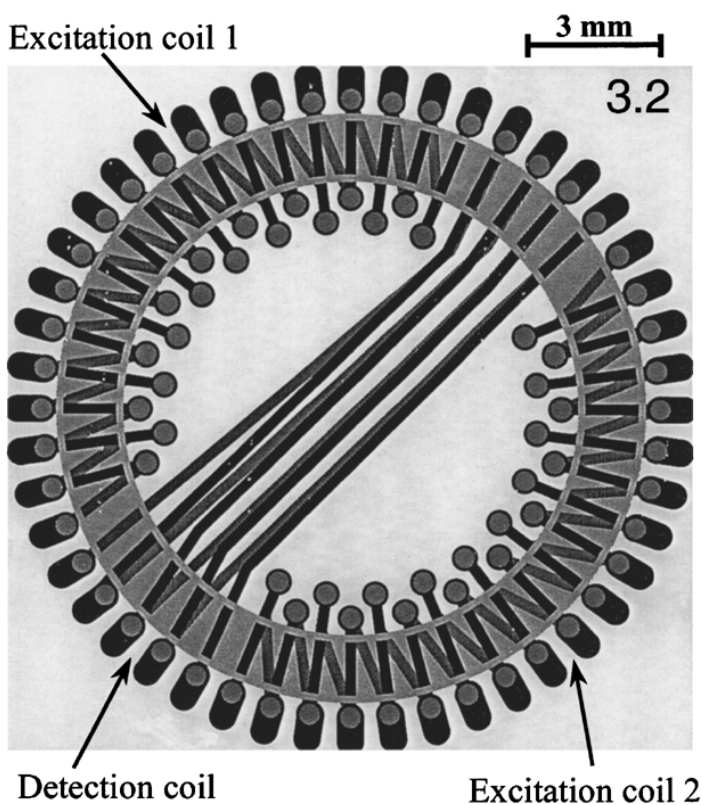

(a)

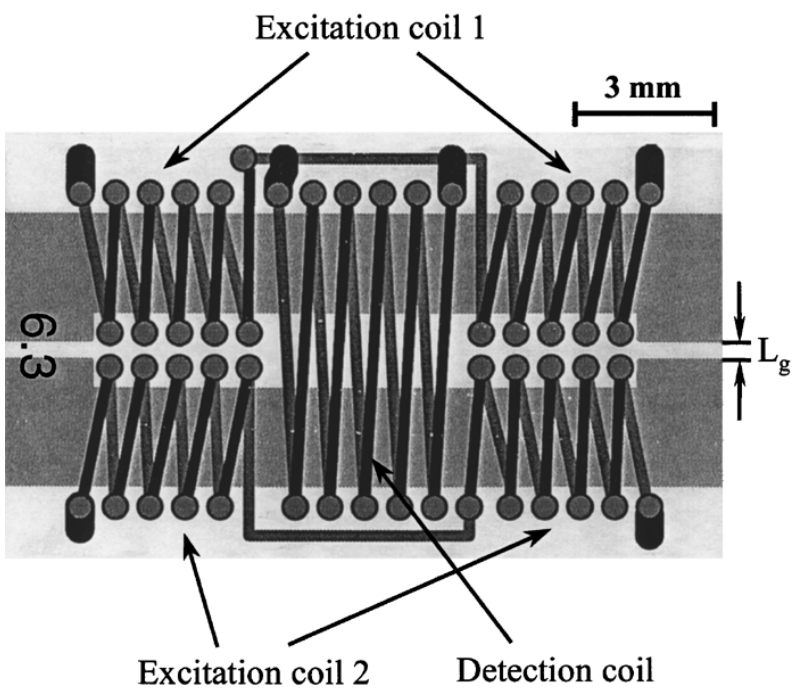

(b)

Fig. 1. Schematic diagram of (a) a toroidal and (b) a rectangular fluxgate sensor showing air gaps with length $L_{g}$.

and then processing according to manufacturer's specifications after pattern transfer. A wet chemical process was developed in-house to etch the magnetic foils, consisting of exposure of the resist-covered foils to a freshly prepared aqua regia type etching bath $\left(\mathrm{HNO}_{3}: \mathrm{HCl}: \mathrm{H}_{2} \mathrm{O}, 2: 1: 3\right)$ for several minutes at room temperature without agitation. The photoresist proved itself immune to the etchant and exhibited excellent adhesion if the substrates were well cleaned.

To align the PCB boards, a pinning system was used. The precision of the pin alignment holes define the maximum lateral precision available with this technology (currently about $100 \mu \mathrm{m}$ ). Bonding operations were carried out by hot pressing typically at $180{ }^{\circ} \mathrm{C}$ and $20 \mathrm{~kg} / \mathrm{cm}^{2}$. During the bonding step, the Prepreg(r) sheets melt, whereby the viscous liquid spreads out and contacts all interior surfaces under the applied pressure. 


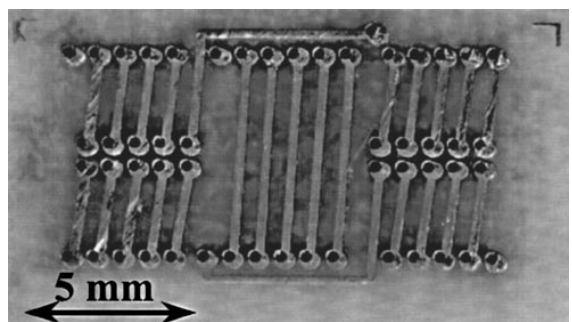

(a)

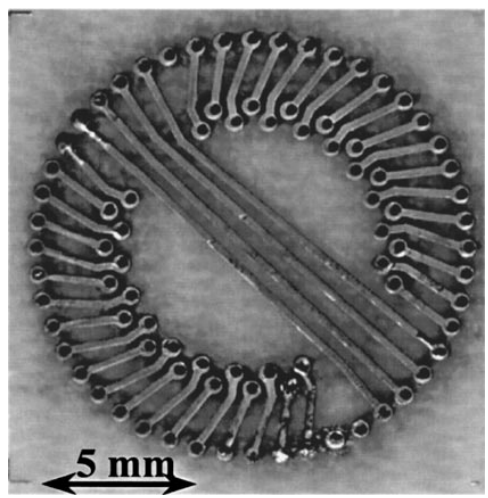

(b)

Fig. 2. Photographs of as-fabricated fluxgate sensors: (a) a toroidal fluxgate and (b) a rectangular fluxgate sensor.

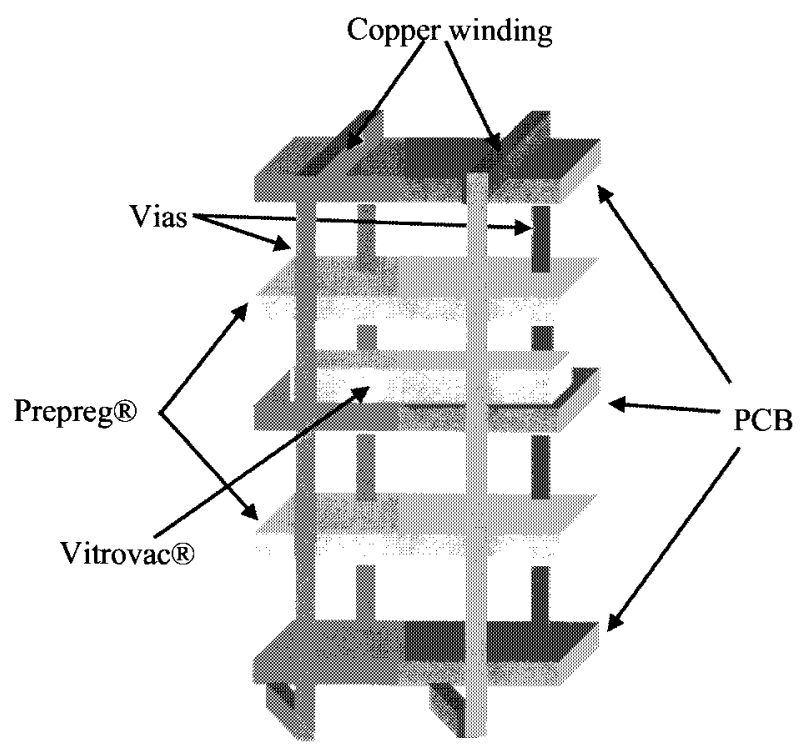

Fig. 3. Schematic diagram of fluxgate fabrication process.

Via holes were drilled at appropriate positions for the interconnects, which were subsequently metallized using copper electroplating. Winding patterns were produced on the outer copper layers photolithographically, thereby completing the helical coil pattern which fully encircles the interior magnetic core.

\section{MEASUREMENTS}

The excitation coils were fed with an ac excitation current of 100-500 $\mathrm{mA}_{\mathrm{p}-\mathrm{p}}$ provided by an HP 6825A bipolar power supply at frequencies of $10-30 \mathrm{kHz}$. The fluxgate response to an external magnetic field $B_{\text {ext }}$ was monitored by connecting the detection coil to a EG\&G 7260 lockin amplifier tuned to the second harmonic frequency $2 f$. Computer controlled measurements of the second harmonic voltage $V_{2 f}$ were made as a function of the external field $B_{\text {ext }}$ generated by Helmoltz coils placed symmetrically around the fluxgate device. These coils were fed with a dc current provided by a programmable Keithley 2400 power supply. The sensitivity $S_{B}=\left(d V_{2 f} / \mathrm{dB}_{\mathrm{ext}}\right)$ was determined by numerical derivation.

\section{EXPERIMENTAL RESULTS AND DISCUSSION}

\section{A. Time-Dependent Response}

In Fig. 4, a series of time-dependent currents and voltages for a rectangular fluxgate magnetic sensor are shown. In particular we will use this figure to explain in a simple way the operation principle of the fluxgate. Fig. 4(a) represents the sinewave excitation current $I_{e}\left(300 \mathrm{~mA}_{\mathrm{p}-\mathrm{p}}\right)$ fed into the two excitation coils. The magnitude of this current was adjusted to alternatively saturate the core in both magnetization directions. Without any external magnetic field $\left(B_{\text {ext }}=0\right)$, zero magnetic flux would be measured by the detection coil because of the two identical but opposite components of the generated flux passing through this coil. When a static magnetic field is applied externally $\left(B_{\text {ext }} \neq 0\right)$, an additional magnetic component will appear in the core, which will add to the excitation flux, and the total time-dependent magnetic field will no longer be equal in magnitude for both sections of the ferromagnetic material.

We will show how the time-dependant flux in the detection coil is composed of contributions, mainly arising from the two ferromagnetic material sections.

In Fig. 4(b) we show the induced voltage $V_{i, 1}=(d \Phi / d t)$ in the first excitation coil, when applying a current of 300 $\mathrm{mA}_{\mathrm{p}-\mathrm{p}}$ in the second excitation coil in the presence of a $B_{\text {ext }}=300 \mu \mathrm{T}$. This $d \Phi / d t$ also represents the time variation of the magnetic flux in one section of the ferromagnetic material within the detection coil under normal operation of the fluxgate. The nonsinusoidal peaked behavior of $V_{i, 1}$ is due to the saturation of the ferromagnetic material. As a result, this signal has a strong component at the second harmonic of the excitation frequency. For the same $B_{\text {ext }}=300 \mu \mathrm{T}$, we show in Fig. 4(c) the induced voltage $V_{i, 2}$ in the second excitation coil, when applying the current of $300 \mathrm{~mA}_{\mathrm{p}-\mathrm{p}}$ in the first excitation coil. The phase difference between $V_{i, 1}$ and $V_{i, 2}$ arises from opposing magnetizations induced by the excitation current and their relative orientation with respect to $B_{\text {ext }}$.

Fig. 4(d) shows the time-dependent voltage $V_{d}$ of the detection coil in the presence of $B_{\text {ext }}=300 \mu \mathrm{T}$. This signal can be thought of as the summation of the contributions of timedependent flux in each of the two sections of the ferromagnetic material within the detection coil, i.e., the summation of $V_{i, 1}$ and $V_{i, 2}$. The latter quantity $\left(V_{i, 1}+V_{i, 2}\right)$ indeed closely resembles the signal $V_{d}$ of Fig. 4(d). One notes the presence of a very strong second harmonic component in $V_{d}$. It is exactly this component which will be used for detecting the 


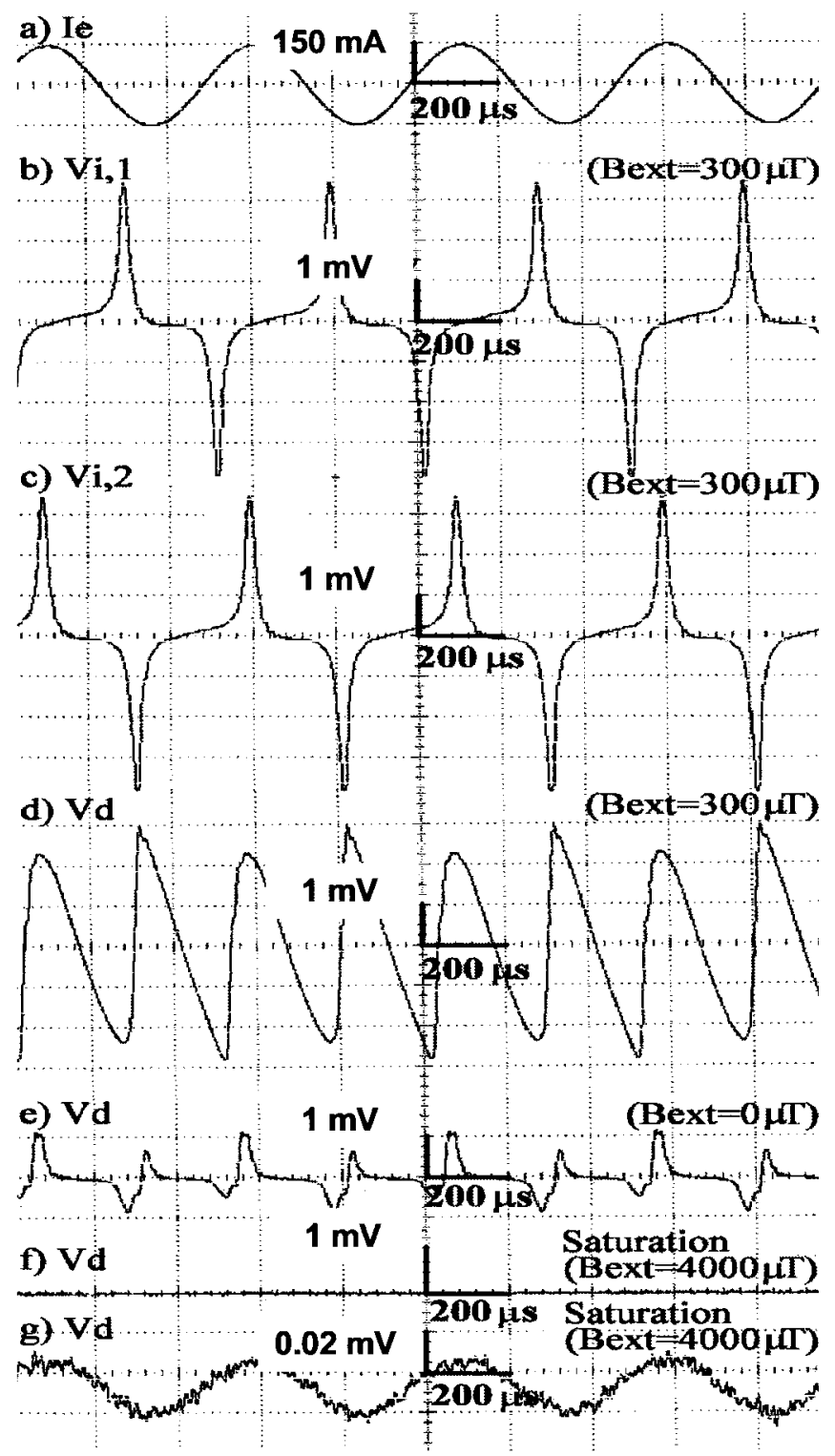

Fig. 4. Time-dependent response: (a) sinewave excitation current $I_{e}$; (b) induced voltage $V_{i, 1}=(d \Phi / d t)$ in the first excitation coil, when applying a current of $300 \mathrm{~mA}$ in the second excitation coil in the presence of a $B_{\text {ext }}=300 \mu \mathrm{T} ;$ (c) induced voltage $V_{i, 2}=(d \Phi / d t)$ in the second excitation coil, for the same choice of parameters as Fig. 4(b); (d) time-dependent voltage of the detection coil $V_{d}$ in the presence of $B_{\text {ext }}=300 \mu \mathrm{T}$; (e) time dependent voltage $V_{d}$ of the of the detection coil at zero external field; (f) time-dependent voltage $V_{d}$ of the detection coil at a very high field $B_{\text {ext }}=4000 \mu \mathrm{T} ;(\mathrm{g})$ zoom of the curve in (f).

magnetic field $B_{\text {ext }}$ with the fluxgate. Fig. 4(e) represents the detection coil signal $V_{d}$ at zero external field. Ideally this signal would be zero when the opposing fluxes in both sections of the ferromagnetic material within the detection coil mutually cancel. However, small geometrical asymmetries in the electrical winding patterns of the coils and magnetic hysteresis effects or the influence of the earth's magnetic field may cause a nonzero response. In any case, the signal amplitude is strongly reduced with respect to the curve of Fig. 4(d). Fig. 4(f) shows the detection coil signal $V_{d}$ at a very high field $B_{\text {ext }}=4000 \mu \mathrm{T}$. This field completely saturates the ferromagnetic material in one direction and therefore the

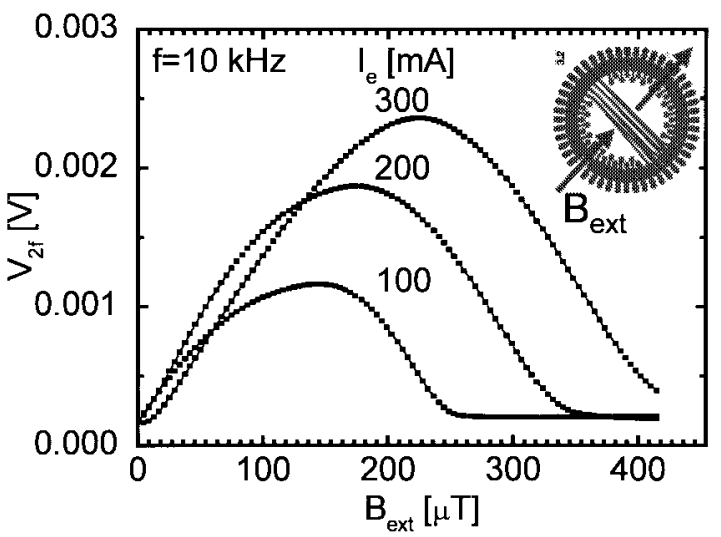

(a)

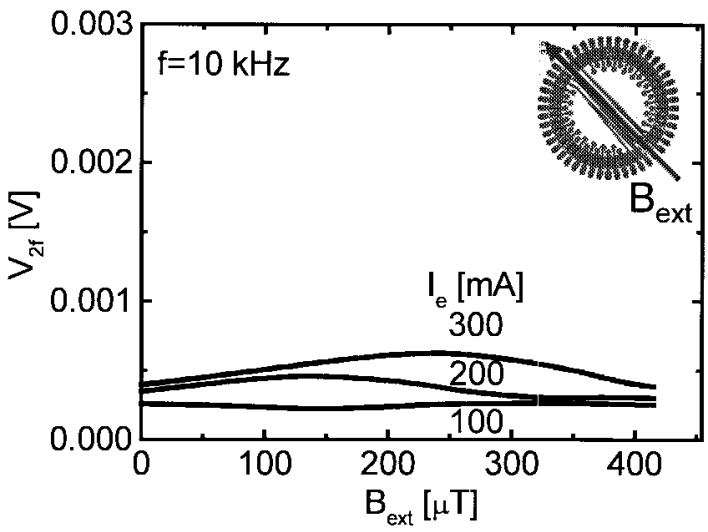

(b)

Fig. 5. Dependence on the orientation of the external field: (a) second harmonic voltage $V_{2 f}$ of the detection coil of a toroidal fluxgate sensor as a function of $B_{\text {ext }}$ for various excitation currents $I_{e}$ at $10 \mathrm{kHz}$; (b) second harmonic voltage $V_{2} f$ for the same fluxgate sensor and for the same choice of parameters as in (a), but for $B_{\text {ext }}$ applied parallel to the windings of the detection coil.

time-dependent response is nearly zero. Fig. $4(\mathrm{~g})$ is a zoom of the curve of Fig. 4(f), showing the presence of a small first harmonic voltage. Generally, one can say that the fluxgate sensor is capable of detecting external magnetic fields, which are in the range of the magnetic field induced via the excitation coil. When $B_{\text {ext }}$ is much smaller or much larger, the fluxgate response will decrease.

\section{B. Dependence on the Orientation of the External Field}

Fig. 5(a) shows the second harmonic voltage $V_{2 f}$ of the detection coil of a toroïdal fluxgate sensor as a function of $B_{\text {ext }}$ for various excitation currents $I_{e}$ at a frequency $f$ of 10 kHz. $B_{\text {ext }}$ is applied in-plane perpendicular to the detection coil, as shown schematically in the insert of Fig. 5(a). For $I_{e}=100 \mathrm{~mA}$, one notes a linear slope at small $B_{\text {ext }}$ and a maximum around $B_{\text {ext }}=150 \mu \mathrm{T}$. Above $250 \mu \mathrm{T}, V_{2 f}$ approaches zero. For values of $I_{e}$ between 200 and $300 \mathrm{~mA}$, the linear slope, the response maximum, and the decrease of $V_{2 f}$ all are shifted to higher $B_{\text {ext }}$. This graph clearly shows that by judiciously choosing the excitation current $I_{e}$, one can select the magnetic field sensitivity range and the linear part of the $V_{2 f}-B_{\text {ext }}$ curve. 


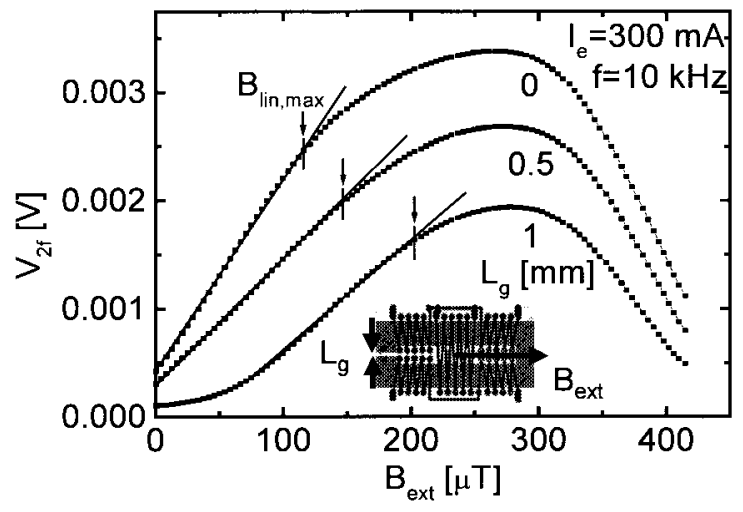

(a)

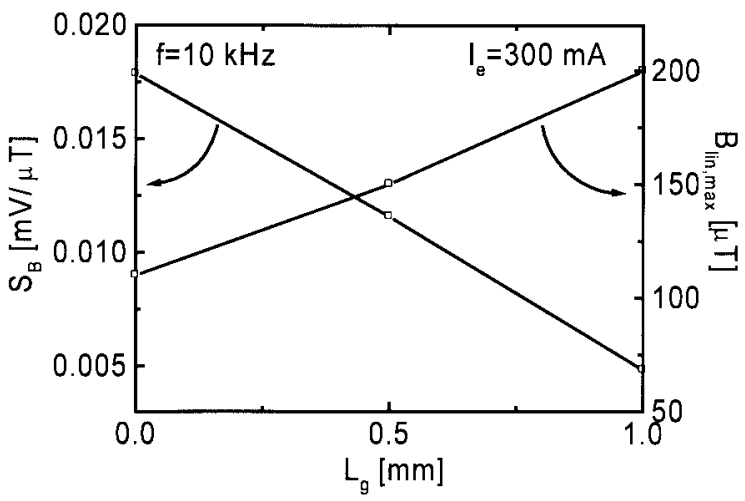

(b)

Fig. 6. Dependence on the air gap: (a) second harmonic voltage $V_{2} f$ of the detection coil of a rectangular fluxgate sensor as a function of $B_{\text {ext }}$ for various gap length $L_{g}$ at $10 \mathrm{kHz}$ and for an excitation current of $300 \mathrm{~mA}$; (b) sensitivity $S_{B}=\left(d V_{2 f} / d B_{\text {ext }}\right)$ in the linear field range and maximum of the linear range $B_{\text {lin,max }}$, determined from the curves of Fig. 6(a).

Fig. 5(b) shows the second harmonic voltage $V_{2 f}$ for the same fluxgate sensor and for the same choice of parameters, except for the fact that $B_{\text {ext }}$ is applied parallel to the windings of the detection coil, as shown schematically in the insert. In this case, the external field does not directly cause an asymmetry within the detection coil. As a result, the response $V_{2 f}$ has strongly decreased. Fig. 5(a) and (b) both clearly show the potential of this fluxgate device for application as a magnetic compass.

\section{Dependence on the Air Gap}

Fig. 6(a) represents the second harmonic voltage $V_{2 f}$ of the detection coil of a rectangular fluxgate sensor as a function of Bext for various gap lengths $L_{g}$ at a frequency of 10 $\mathrm{kHz}$ and for an excitation current of $300 \mathrm{~mA}_{\mathrm{p}^{-} \mathrm{p}}$. $B_{\text {ext }}$ is applied in-plane perpendicular to the detection coil, as shown schematically in the insert of Fig. 6(a). The three curves are characterized by a linear slope at low $B_{\text {ext. }}$. For $L_{g}=0$ $\mathrm{mm}$, one notes a maximum field, limiting this linear range of $B_{\text {lin, } \max } \approx 110 \mu \mathrm{T}$, while the maximum value of $V_{2 f}$ is around $280 \mu \mathrm{T}$. For $L_{g}=0.5$ and $1 \mathrm{~mm}$, the maxima of the linear ranges $B_{\text {lin, } \max }$ are shifted to higher $B_{\text {ext }}$, while the maximum of the curves is found at the same external field $B_{\text {ext }}=280 \mu \mathrm{T}$ in both cases. In other words, the slopes of the linear parts of the $V_{2 f}-B_{\text {ext }}$ curves decrease with the

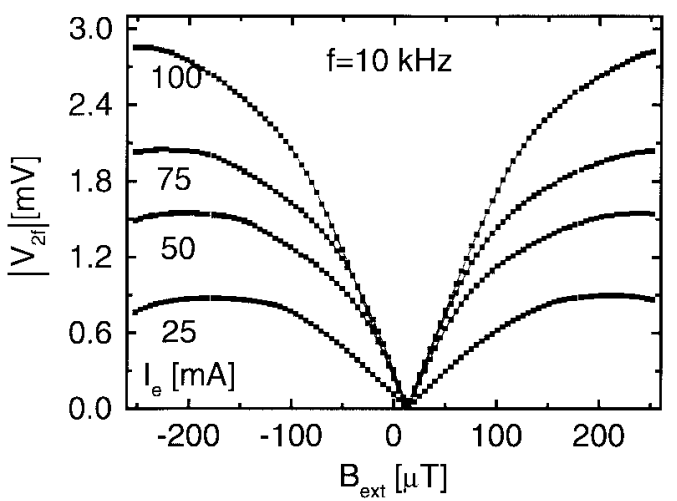

Fig. 7. Dependence on the excitation current: second harmonic voltage $V_{2} f$ (plotted as the absolute value) of the detection coil of a rectangular fluxgate sensor without air gap $\left(L_{g}=0 \mathrm{~mm}\right)$ as a function of $B_{\text {ext }}$ for various excitation currents $I_{e}$ at $10 \mathrm{kHz}$.

increasing gap length $L_{g}$. This graph clearly shows that by choosing a certain air gap $L_{g}$, one can select the fluxgate sensor's sensitivity and the linear magnetic field range.

Fig. 6(b) shows the sensitivity in the linear field range of the fluxgate and the maximum of the linear range $B_{\text {lin,max }}$, determined from the curves of Fig. 6(a). One notes a decrease of the sensitivity $\mathrm{S}_{B}$ and an increase of the maximum of the linear range $\mathrm{B}_{1}$ in, $\max$ with increasing gap length $L_{g}$. It is seen from Fig. 6 that by choosing a certain air gap $L_{g}$, there is a compromise to be made between a good sensitivity and a wide magnetic field range.

Kawahito et al. [4] have shown that upon excitation of the fluxgate sensor by an ac magnetic field with triangular waveform, the Fourier analysis of the induced voltage in the detection coil gives rise to a second harmonic voltage $V_{2 f}$ given by

$$
V_{2 f}=\frac{8 N A \mu_{r}^{*} B_{m} f}{\pi} \sin \frac{\pi \Delta B}{B_{m}} \sin \frac{\pi B_{\mathrm{ext}}}{B_{m}}
$$

where $N$ is the number of turns of the detection coil, $A$ is the cross-section of the area of the core, $\mu_{r}^{*}$ the effective relative permeability, and $B_{m}$ the maximum magnetic induction generated by the excitation current. $\Delta B=2 B_{s} / \mu_{r}^{*}\left(B_{s}\right.$ is the saturation magnetic induction). Under the optimal excitation condition where the peak excitation field is twice the saturation field, Kawahito et al. derive, starting from (1), a magnetic sensitivity of the fluxgate sensor

$$
S_{B}[V / T]=d V_{2 f} / d B_{\text {ext }}=8 N A \mu_{r}^{*} f .
$$

Equation (2) clearly shows the linear dependence on the various characteristic parameters of the fluxgate sensor. In particular the influence of the effective relative permeability $\mu_{r}^{*}$ that decrease with increasing air gap length $L_{g}$.

\section{Dependence on the Excitation Current}

Fig. 7 represents the second harmonic voltage $V_{2 f}$ of the detection coil of a rectangular fluxgate sensor without air gap $\left(L_{g}=0 \mathrm{~mm}\right)$ as a function of $B_{\text {ext }}$ for various excitation currents $I_{e}$ at $10 \mathrm{kHz}$. The relative orientation of $B_{\text {ext }}$ is as shown in the insert of Fig. 6(a). We have chosen the direction of $B_{\text {ext }}$ in the line of the horizontal component of the local 


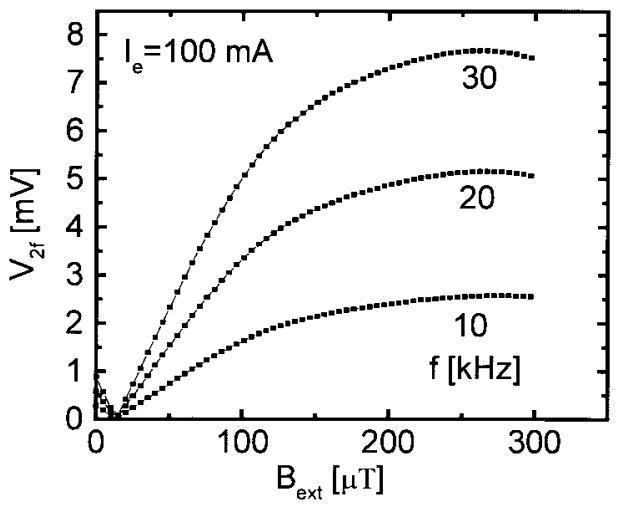

(a)

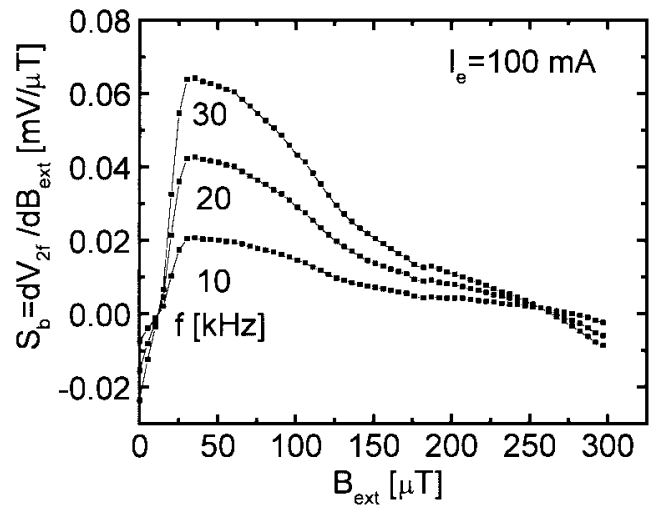

(b)

Fig. 8. Dependence on the frequency of the excitation current: (a) second harmonic voltage $V_{2} f$ of the detection coil of a rectangular fluxgate sensor without air gap $L_{g}=0 \mathrm{~mm}$ as a function of $B_{\text {ext }}$ for various frequency of the excitation current $I_{e}=100 \mathrm{~mA}$; (b) sensitivity $S_{B}=\left(d V_{2 f} / d B_{\text {ext }}\right)$ determined by numerical derivation from the curves of Fig. 8(a).

terrestrial magnetic field (in Switzerland $B_{\text {terestrial }} \approx 20 \mu \mathrm{T}$ ). One notes that for all excitation currents, $V_{2 f}$ approaches zero at this field; this situation corresponds to an effective zero magnetic field. One notes that for all $I_{e}$, there is a linear dependence on $B_{\text {ext }}$ in the low field range. This range becomes more extended for the higher excitation currents. The symmetric response around the true zero field with respect to the sign of the external magnetic field is immediately clear. Moreover, the curves shown in Fig. 7 represents data taken by increasing the external field from $-250 \mu \mathrm{T}$ up to $250 \mu \mathrm{T}$ and vice versa. Within the limit of our detection, we found no evidence of any hysteretic behavior.

\section{E. Dependence on the Frequency of the Excitation Current}

Fig. 8(a) represents the second harmonic voltage $V_{2 f}$ of the detection coil of a rectangular fluxgate sensor without air gap $L_{g}=0 \mathrm{~mm}$ as a function of $B_{\text {ext }}$ for various frequencies of the excitation current $\left(I_{e}=100 \mathrm{~mA}_{\mathrm{p}-\mathrm{p}}\right)$. The relative orientations are the same as in the insert of Fig. 6(a). Like in Fig. 7, the zero response of $V_{2 f}$ for $B_{\text {ext }}=20 \mu \mathrm{T}$ is characteristic of the earth's magnetic field. The three curves are characterized by a linear slope at low $B_{\text {ext }}$. At $30 \mathrm{kHz}$, one notes a maximum value of $V_{2 f}$ around $300 \mu \mathrm{T}$ and a relatively large slope for the linear part of the curve. At lower frequencies of 20 and 10

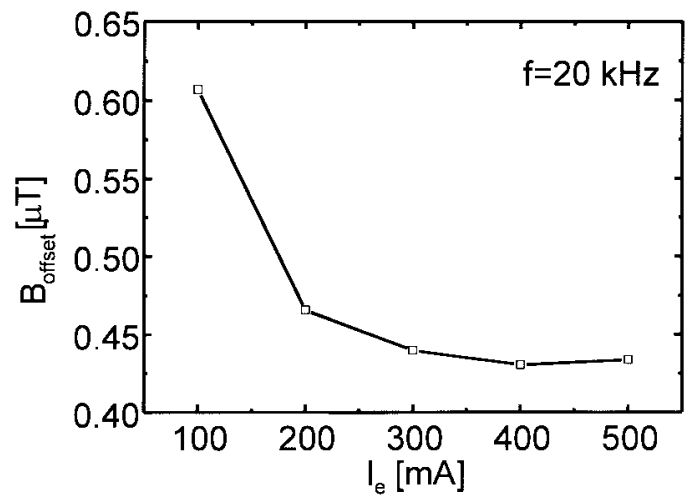

Fig. 9. Magnetic offset $B_{\text {offset }}$ of the fluxgate sensor as a function of excitation current $I_{e}$ at $20 \mathrm{kHz}$ for the rectangular fluxgate without gap.

$\mathrm{kHz}$, there is a progressive decrease in both the slopes of the linear parts and in the response maxima.

Fig. 8(b) shows the sensitivity $S_{B}=\left(d V_{2 f} / d B_{\text {ext }}\right)$ determined from the curves of Fig. 8(a). One observes a plateau in the sensitivity of about $0.06 \mathrm{mV} / \mu \mathrm{T}$ up to $B_{\text {ext }} \approx 80 \mu \mathrm{T}$ for the highest excitation frequency $(30 \mathrm{kHz})$. For lower frequencies, there is a decrease of the sensitivity value, but the plateau field range is not changed significantly, indicating a frequency-independent $\mu_{r}^{*}\left(B_{\text {ext }}\right)$ behavior in this frequency range. Taking the experimental value $\left(S_{B}=60 \mathrm{~V} / \mathrm{T}\right)$ we can estimate from (2) a value for $\mu_{r}^{*}=2000$, clearly indicating that with our devices we are not yet under the optimal operation conditions. The reason may be the relative wide spacing between the electrical windings around the Vitrovac ${ }^{\circledR}$ core resulting in a substantial loss of flux in the dielectric around the winding pattern [10].

When normalizing the sensitivities obtained by the authors mentioned in the introduction of this article, to the same frequency $(f=30 \mathrm{kHz})$ and to the same winding number $(N=5)$, our measured sensitivity, derived from Fig. 8(a) $\left(S_{B}=60 \mathrm{~V} / \mathrm{T}\right)$ intrinsically can be a factor of 50 higher than the results of [4] and [6] and a factor $4 / 3$ higher than the result of [8]. The fluxgate sensors presented here show that they are suitable for high performance applications. The key factor determining the high sensitivity of our fluxgate devices is the extremely high relative permeability of the Vitrovac $\AA$ magnetic foil material.

\section{F. Magnetic Offset of the Fluxgate Sensor}

To evaluate the minimum detection limit of our fluxgate sensing devices, we have performed measurements of the magnetic offset of the fluxgate sensor $B_{\text {offset. Initializing }}$ our devices by a negative saturation field, we hereafter have measured the voltage $V_{2 f}$ for a rectangular fluxgate, when cycling $B_{\text {ext }}$ from zero to saturation and back to zero. From the two values of $V_{2 f}$, obtained by approaching zero both from a negative and positive saturation field, one can calculate the offset by using the fluxgate's measured sensitivity curves. The results of such procedure are shown in Fig. 9 as a function of excitation current $I_{e}$ at $20 \mathrm{kHz}$. One observes a decreasing $B_{\text {offset }}$, i.e., an improved minimum field detection limit, with increasing $I_{e}$. The reason for this behavior has to be sought in 
the better defined magnetic saturation state at high applied $I_{e}$ (smaller width of magnetic hysteresis curve at high $I_{e}$ ). The observed values of $B_{\text {offset }}$ for our fluxgate sensor clearly are well below the value of the earth's magnetic field, enabling application of our devices for example as a magnetic compass.

\section{CONCLUSIONS}

The proposed hybrid technology presented in this paper is capable of producing fluxgate sensors with performance characteristics that are comparable to or surpass those of previously reported miniature fluxgate devices. The second harmonic voltage $V_{2 f}$ of the detection coil of a toroidal fluxgate sensor has been studied as a function of the direction of the external field $B_{\text {ext }}$ (parallel or perpendicular to the windings of the detection coil). This experiment clearly shows the potential of this fluxgate device for application as a magnetic compass. The $V_{2 f}$ response of rectangular fluxgate sensors has also been studied as a function of the gap length, excitation current, and frequency. By choosing one, or a combination of these parameters, we demonstrated that the fluxgate sensor's sensitivity and the linear magnetic field range can be selected and optimized.

This new hybrid PCB/magnetic metal foil technology permits, in comparison with time consuming and expensive thin film deposition techniques, the facile and relatively inexpensive realization of high sensitivity $(60 \mathrm{~V} / \mathrm{T}$ at $30 \mathrm{kHz}$ for a five windings detection coil wound around a rectangular magnetic core) fluxgate sensors. The cost aspects, mass production capabilities, and high degree of flexibility offered by the choice of different magnetic materials are reasons to propose this new hybrid technology as a serious alternative to the currently available micromachined fluxgate devices.

\section{ACKNOWLEDGMENT}

The authors would like to thank L. Chiesi and B. Janossy of the Institute of Microsystems for their assistance in the fluxgate measurements, and M. Hermanjat and P. Vossler of the Department of Electrical Engineering at EPFL for their help in the process conception.

\section{REFERENCES}

[1] F. Primdahl, "The fluxgate magnetometer," J. Phys. E: Sci. Instrum., vol. 12, pp. 241-253, 1979.

[2] P. Ripka, "Review of magnetic fluxgate sensor," Sens. Actuators A, vol. A33, pp. 129-134, 1992.

[3] N. Pollock, "Electronic compass using a fluxgate sensor," Wireless World, 1982, pp. 49-54.

[4] S. Kawahito, Y. Sasaki, H. Sato, T. Nakamura, and Y. Tadokoro, "A fluxgate magnetic sensor with micro-solenoids and electroplated permalloy cores," Sens. Actuators A, vol. A43, pp. 128-134, 1994.

[5] T. Seitz, "Fluxgate sensor in planar microtechnology," Sens. Actuators A, vol. A21-23, pp. 799-802, 1990.

[6] S. O. Choi, S. Kawahito, Y. Matsumoto, M. Ishida, and Y. Tadokoro, "An integrated micro fluxgate magnetic sensor," Sens. Actuators A, vol. A55, pp. 121-127, 1996
[7] S. Kawahito, H. Satoh, M. Sutoh, and Y. Tadokoro, "High-resolution micro-fluxgate sensing elements using closely coupled coil structures," Sens. Actuators A, vol. A54, pp. 612-617, 1996.

[8] R. Gottfried-Gottfried, W. Budde, R. Jähne, H. Kück, B. Sauer, S. Ulbricht, and U. Wende, "A miniaturized magnetic-field sensor system consisting of a planar fluxgate sensor and a CMOS readout circuitry," Sens. Actuators A, vol. A54, pp. 443-447, 1996.

[9] P. Ripka, M. Tondra, J. Stokes, and R. Beech, "AC-driven AMR and GMR magnetoresistors," in Proc. 12th Eur. Conf. Solid-State Transducers, Eurosensors XII, N. M. White, Ed. Southampton, U.K.: Inst. Physics Publ., 1998.

[10] O. Dezuari, S. E. Gilbert, E. Belloy, and M. A. M. Gijs, "A new hybrid technology for planar microtransformer fabrication," Sens. Actuators A, vol. 71/3, pp. 198-207, 1998.

Olivier Dezuari was born in Lausanne, Switzerland, in 1972. He received the diploma in microengineering from the Swiss Federal Institute of Technology, (EPFL), Lausanne, Switzerland, in 1997.

In September 1997, he joined the Institute of Microsystems as Research Assistant in the field of developing new technologies for the fabrication of inductive components, in particular flexible components.

Eric Belloy was born in Geneva, Switzerland, in 1972. He received the diploma in microengineering in 1997 from the Swiss Federal Institute of Technology (EPFL), Lausanne, Switzerland.

Since April 1997, he has been with he Institute of Microsystems at EPFL as a Research Assistant, where he is developing a new technology based on flex-foil patterning and assembling, in order to realize magnetic devices.

Scott E. Gilbert received the Ph.D. degree in physical chemistry from the University of California, Santa Barbara, in 1993.

He then came immediately to the Swiss Federal Institute of Technology in Lausanne (EPFL) to postdoc at the Institute of Physical Chemistry for work on nanostructured liquid junction photovoltaic devices. Afterwards, he moved to the Department of Physics where his interests expanded to explore novel methods for ultra-thin metallic film and magnetic nanowire electrodeposition and electrical contacting methods. He is now with Prof. Gijs in the Institute of Microsystems as Project Leader for the development of new technologies for miniature inductive devices and electrochemical micromachining.

Martin A. M. Gijs received the degree in physics in 1981 and the Ph.D degree in physics in 1986 from the Katholieke Universiteit Leuven, Belgium.

He joined the Philips Research Laboratories in Eindhoven, The Netherlands, in 1987. Subsequently, he has worked there on micro-and nano-fabrication processes of high critical temperature superconducting Josephson and tunnel junctions, the microfabrication of microstructures in magnetic multilayers showing the giant magnetoresistance effect, the design and realization of miniaturized motors for hard disk applications, and the design and realization of planar transformers for miniaturized power applications. He joined the Swiss Federal Institute of Technology Lausanne (Ecole Polytechnique Fédérale de Lausanne) in 1997 as a Professor in the Institute of Microsystems of the Microengineering Department, where he is responsible for the Microsystems Technology Group. His main interests are in developing technologies for novel inductive-type devices, new microfabrication technologies for microsystems fabrication in general, and the development and use of microsystems technologies for biomedical applications in particular. 